\title{
Process and parameter optimisation for micro structuring of 3D freeform metallic surfaces: a comparative study of short-pulse (nanosecond) and ultrafast (picosecond, femtosecond) laser ablation
}

Steffen G. Scholz, Daniel J. B. S. Sampaio, Melanie Mangang, Wilhelm Pfleging

Steffen G. Scholz, Daniel J. B. S. Sampaio, Melanie Mangang, Wilhelm Pfleging, "Process and parameter optimisation for micro structuring of 3D freeform metallic surfaces: a comparative study of short-pulse (nanosecond) and ultrafast (picosecond, femtosecond) laser ablation," Proc. SPIE 10092, Laser-based Micro- and Nanoprocessing XI, 100920E (17 February 2017); doi: 10.1117/12.2252044

SPIE. Event: SPIE LASE, 2017, San Francisco, California, United States 


\title{
Process and parameter optimisation for micro structuring of $\mathbf{3 d}$ freeform metallic surfaces - a comparative study of short-pulse (nanosecond) and ultrafast (picosecond, femtosecond) laser ablation
}

\author{
Steffen G. Scholz ${ }^{\mathrm{a}}$, Daniel J. B. S. Sampaio ${ }^{\mathrm{a}, \mathrm{d}}$, Melanie Mangang ${ }^{\mathrm{b}}$, Wilhelm Pfleging, \\ ${ }^{a}$ Karlsruhe Institute of Technology, Institute for Applied Computer Science (IAI), H.-von- \\ Helmholtz-Platz, 76344 Egg.-Leopoldshafen, Germany; ${ }^{b}$ Karlsruhe Institute of Technology, Institute \\ for Applied Materials (IAM - AWP), H.-von-Helmholtz-Platz, 76344 Egg.-Leopoldshafen, \\ Germany; 'Karlsruhe Nano Micro Facility, H.-von-Helmholtz-Platz 1, 76344 Egg.-Leopoldshafen, \\ Germany; 'Universidade Estadual Paulista (Unesp), Faculdade de Engenharia, Av. Ariberto P. da \\ Cunha 333, 12516410 Guaratinguetá-SP, Brazil
}

\begin{abstract}
Layer-based laser ablation of three dimensional micro structured freeform surfaces has become of significant importance for technical applications such as biomimetic surfaces in recent years. In order to identify the optimum set of process parameters for a complex laser ablation operation, a design of experiments (DoE) study has been carried out with laser sources covering pulse durations regime of femtosecond (fs), picosecond (ps) and nanosecond (ns). The aim was to identify the optimum parameter set for achieving best surface roughness and, as a second criteria, for machining time to be reduced to a minimum. In a first step, rectangular pockets have been machined and a DoE based parameter variation was performed. In particular, the parameters wavelength $(1030 \mathrm{~nm}, 515 \mathrm{~nm}, 343 \mathrm{~nm})$, machining speed, laser power, and laser pulse duration (fs, ps, ns) have been modified. Surface roughness and ablated depth were measured and an optimum set of parameters was calculated. The results show that the ultraviolet laser type $(343 \mathrm{~nm})$ has the best performance to achieve lowest surface roughness and with a laser pulse duration of 3445 fs reaches also the best ablation efficiency in relation to machining time. While machining speed and laser power have an almost linear influence on achievable roughness, laser pulse duration has a quadratic influence in relation to a global minimum on the surface roughness result. For the ablated depth, machining speed and laser power have an almost linear influence while laser pulse duration has a quadratic influence in relation to a global maximum.
\end{abstract}

Keywords: Process optimisation, laser ablation, ablation regime, ultrashort pulse laser machining.

\section{INTRODUCTION}

Lasers have been used for many years in industrial material processing applications by means of its concentrated energy for ablating, drilling, cutting, welding, and other alterations of the material. The advantages of laser processing over other methods are, among others, the reduced heat-affected zone, speed, versatility, and environmental concerns. The noncontact nature of laser machining applications, as drilling and cutting, also eliminates tool wear which is sometimes a problem in traditional machining [1-3]. These advantages of ultrashort laser pulses have been applied in precision micromachining of various materials. Several laser machines were developed for material removal, varying on the laser pulse duration and some material specific time parameters [4-5]. The material dependent time parameters, electron cooling time $\left(\tau_{\mathrm{e}}\right)$, lattice heating time $\left(\tau_{\mathrm{i}}\right)$ and laser pulse duration $\left(\tau_{\mathrm{L}}\right)$ are considered important. As a rule, $\tau_{\mathrm{e}}<<\tau_{\mathrm{i}}$ and, for most materials, $\tau_{\mathrm{i}}$ is in the picosecond range. According to the laser pulse length, three different ablation regimes are defined, been femtosecond pulses if $\tau_{\mathrm{L}}<\tau_{\mathrm{e}}<\tau_{\mathrm{i}}$, picosecond pulses if $\tau_{\mathrm{e}}<\tau_{\mathrm{L}}<\tau_{\mathrm{i}}$ and nanosecond pulses if $\tau_{\mathrm{e}}<\tau_{\mathrm{i}}<\tau_{\mathrm{L}}$.

During the laser pulse, the energy is absorbed by the top surface layer of the substrate where heat is generated and the material starts melting, raising to the vaporization temperature. The long pulse duration allows a thermal wave to propagate into the material through conduction and evaporation occurs from the liquid material. The molten material is partially ejected from the cavity by the vapor and plasma pressure, but a part of it remains near the surface, held by surface tension forces. After the pulse, the heat quickly dissipates into the bulk of the material and a recast layer is formed [6-7].

Laser-based Micro- and Nanoprocessing XI, edited by Udo Klotzbach, Kunuhiko Washio, Rainer Kling, Proc. of SPIE Vol. 10092, 100920E · @ 2017 SPIE · CCC code: 0277-786X/17/\$18 · doi: 10.1117/12.2252044 
Besides the laser pulse duration, there are other important parameters influencing the laser ablation process. The first is the wavelength of the laser light used, normally mentioned as Infrared (IR), Green or Ultraviolet (UV) according with the laser light spectrum. Another parameter is the laser peak power that must be large enough to vaporize the material in the laser beam spot [8]. The machining speed in $\mathrm{mm} / \mathrm{s}$ is also an important parameter because defines how long the laser beam intensity will be concentrated in a defined area.

Layer-based laser ablation of three dimensional micro structured freeform surfaces has become of significant importance for technical applications such as biomimetic surfaces in recent years. These biomimetic surfaces with micro and nano scale features provide functional properties that can be used in products to achieve self-cleaning, anti-reflectivity or drag reduction [9]. House fly eye and shark skin like surface structures are promising manufacturing routes for high throughput fabrication of components with micro and nano structured surfaces [10].

It has been shown that the volume ablation rate for metals at different laser pulse-durations from ps to fs increases for shorter pulses [11]. In this work, in order to identify the optimum set of process parameters for a complex laser ablation operation like manufacturing biomimetic surfaces, a design of experiments (DoE) study has been carried out with laser sources covering pulse durations regime of femtosecond ( $\mathrm{fs}$ ), picosecond ( $\mathrm{ps}$ ) and nanosecond (ns). The average roughness $(\mathrm{Ra})$ and the ablation depth were measured and used as quality marks. The optimum set of process parameters should achieve, as a first criteria, the best surface roughness and, as a second criteria, the machining time to be reduced to a minimum.

\section{TOOLS AND DESIGN OF EXPERIMENTS}

\subsection{Laser system}

The laser system used in this investigation was a laser micromachining workstation (SP450-TO, Optec, Belgium) [12], shown in Figure 1(a), equipped with a tunable ultrafast laser (Tangerine, Amplitude SYSTEMES, France) operating with an average power of $20 \mathrm{~W}$ and a maximum pulse energy of $100 \mu \mathrm{J}$ at $1030 \mathrm{~nm}$ wavelength. It has accessible multiwavelength operation in a compact layout with heads for Infrared (1030nm), Green $(515 \mathrm{~nm})$ and Ultraviolet (343 nm), that are mounted on a precision optical turret and can be indexed into position by a mouse click with $\mu \mathrm{m}$ repeatability. A fourth turret position carries zoom microscope and topography probe. The pulse duration can be tuned in the range from 350 fs up to $10 \mathrm{ps}$.

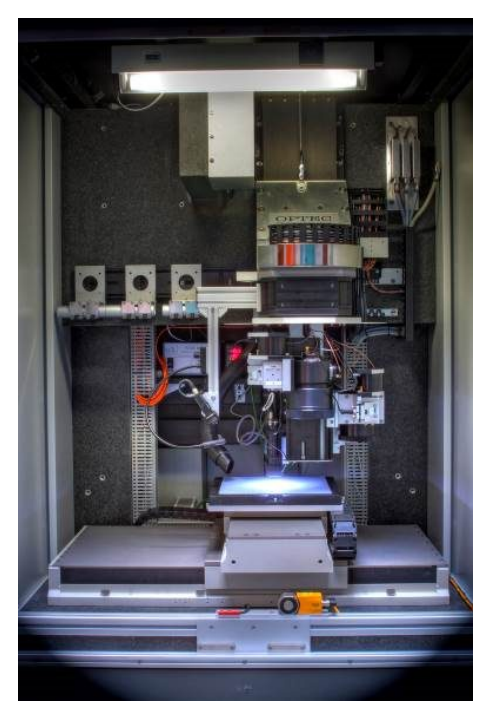

(a)

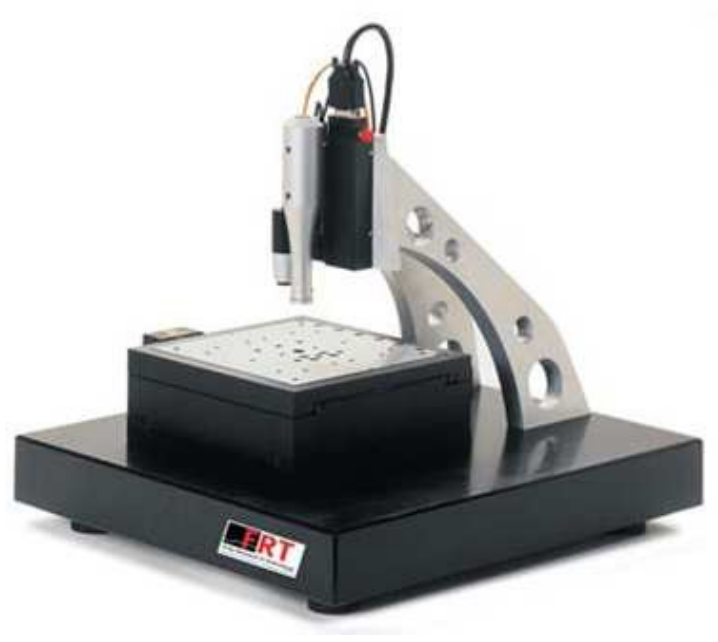

(b)

Figure 1. (a) Micromachining workstation SP450-TO from Optec. (b) Surface metrology tool MicroProf® 100. 


\subsection{Surface metrology system}

The surface measurements in this investigation were made using a universal surface metrology tool (MicroProf ${ }^{\circledR}$ 100, FRT GmbH) [13], shown in Figure 1(b), that is a compact table top based on proven FRT multi-sensor technology, in which different optical measurement methods, which can otherwise only be found in individual solutions, are merged into a universal and space saving device. The equipment software can be individually configured and measurement tasks can be performed either manually or automatically. In this investigation, the roughness (Ra) and the ablation depth were measured in the produced samples.

\subsection{Design of Experiments}

This study in based on a design of experiment (DoE) aiming the understanding of the process parameters, the interaction effect of the independent parameters on the quality marks (roughness and ablation depth) and their optimization. The selected design matrix was a circumscribed three-factor central composite design that requires five levels for each independent parameter. Based on the already mentioned main influences in the laser system, wavelength, laser pulse duration, laser power and machining speed have been selected as independent parameters and their values changed during the experiments according with a defined design matrix.

The laser system available for the tests, as cited, has three different laser lights with the following wavelengths: IR $1030 \mathrm{~nm}$, Green $-515 \mathrm{~nm}$ and UV $-343 \mathrm{~nm}$. The design matrix was then created with the other three parameters: laser pulse duration (fs, ps, ns), laser power and machining speed. Each parameter set trial was repeated three times for each wavelength available. Since five levels are required for each independent parameter remaining, the design matrix resulted in a total of 22 trials and 15 different parameter sets ( 8 trials with the parameter set index 15 of the central point). Table 1 shows the created design matrix for the experiments, presenting for each trial number, the parameter set index and the corresponding parameter values.

Table 1. Design Matrix used to guide the experiments.

\begin{tabular}{ccccc}
\hline $\begin{array}{c}\text { Parameters } \\
\text { for } \\
\text { Trial\# }\end{array}$ & $\begin{array}{c}\text { Laser Pulse } \\
\text { Duration } \\
{[\mathbf{f s}]}\end{array}$ & $\begin{array}{c}\text { Laser } \\
\text { Power } \\
{[\mathbf{W}]}\end{array}$ & $\begin{array}{c}\text { Machining } \\
\text { Speed } \\
{[\mathbf{m m} / \mathbf{s}]}\end{array}$ & $\begin{array}{c}\text { Parameter } \\
\text { Set } \\
\text { Index }\end{array}$ \\
\hline $\mathbf{1}$ & -1 & -1 & -1 & 1 \\
$\mathbf{2}$ & -1 & -1 & +1 & 2 \\
$\mathbf{3}$ & -1 & +1 & -1 & 3 \\
$\mathbf{4}$ & -1 & +1 & +1 & 4 \\
$\mathbf{5}$ & +1 & -1 & -1 & 5 \\
$\mathbf{6}$ & +1 & -1 & +1 & 6 \\
\hline $\mathbf{7}$ & +1 & +1 & -1 & 7 \\
$\mathbf{8}$ & +1 & +1 & +1 & 8 \\
$\mathbf{9}$ & -1.68 & 0 & 0 & 9 \\
$\mathbf{1 0}$ & +1.68 & 0 & 0 & 10 \\
$\mathbf{1 1}$ & 0 & -1.68 & 0 & 11 \\
\hline $\mathbf{1 2}$ & 0 & +1.68 & 0 & 12 \\
$\mathbf{1 3}$ & 0 & 0 & +1.68 & 13 \\
$\mathbf{1 4}$ & 0 & 0 & -1.68 & 14 \\
$\mathbf{1 5}$ & 0 & 0 & 0 & 15 \\
\hline $\mathbf{1 6}$ & 0 & 0 & 0 & 15 \\
$\mathbf{1 7}$ & 0 & 0 & 0 & 15 \\
\hline $\mathbf{1 8}$ & 0 & 0 & 0 & 15 \\
$\mathbf{1 9}$ & 0 & 0 & 0 & 15 \\
$\mathbf{2 0}$ & 0 & 0 & 0 & 15 \\
$\mathbf{2 1}$ & 0 & 0 & 0 & 15 \\
$\mathbf{2 2}$ & 0 & 0 & 0 & 15 \\
\hline & & & & \\
\hline
\end{tabular}




\section{EXECUTION OF THE DOE}

The experiments were executed in rectangular pockets of AISI 316 stainless steel and each experiment was performed in an area of $5 \mathrm{~mm} \times 5 \mathrm{~mm}$. The experimental parameter values had to be adapted for each wavelength characteristics, been the experimental values matrix for each independent parameter in each wavelength showed in Table 2.

Table 2. Experimental parameter values used for each wavelength.

\begin{tabular}{|c|c|c|c|}
\hline Wavelength & Parameter & $\begin{array}{c}\text { Notation } \\
"-1 "\end{array}$ & $\begin{array}{c}\text { Notation } \\
"+1 "\end{array}$ \\
\hline \multirow{3}{*}{$\begin{array}{c}\text { Green } \\
(515 \mathrm{~nm})\end{array}$} & Laser Pulse Duration [fs] & 1253 & 4047 \\
\hline & Laser Power [W] & 3,25 & 4,85 \\
\hline & Machining Speed $[\mathrm{mm} / \mathrm{s}]$ & 210,7 & 799,3 \\
\hline \multirow{3}{*}{$\begin{array}{l}\text { Infrared } \\
(1030 \mathrm{~nm})\end{array}$} & Laser Pulse Duration [fs] & 1253 & 4047 \\
\hline & Laser Power [W] & 5,22 & 8,78 \\
\hline & Machining Speed $[\mathrm{mm} / \mathrm{s}]$ & 218,6 & 801,4 \\
\hline \multirow{3}{*}{$\begin{array}{c}\text { Ultraviolet } \\
\text { (343nm) }\end{array}$} & Laser Pulse Duration [fs] & 1253 & 4047 \\
\hline & Laser Power [W] & 0,304 & 0,376 \\
\hline & Machining Speed $[\mathrm{mm} / \mathrm{s}]$ & 129,6 & 480,4 \\
\hline
\end{tabular}

As mentioned, each experiment was executed three times for each parameter set. After that, the values of roughness and ablated depth were measured and the average value of the three experiments was used as quality mark for both, roughness and ablated depth.

Unfortunately, in the experiments using the green light, for the parameter set for trial\# 14, the machining speed was too slow and the parameter set had to be rejected. In replacement to this experiment, the parameter set index 15 (central point) was once more executed, having this wavelength therefore nine repetitions of the central point. The experiments with the infrared light presented the same issue as the green light for the trial \#14 and also another one with the trial\# 7 , in which the resulting surface was not measurable. In replacement to these experiments, the parameter set index 15 (central point) was twice more executed, having this wavelength therefore ten repetitions of the central point. The experiments using the ultraviolet light had no issue during the execution, following exactly the planned design of experiments.

\section{DATA ANALYSIS AND RESULTS}

The data analysis used the software Matlab (Statistics and Machine Learning Toolbox) [14] and Microsoft Excel. The first analysis was the evaluation of the raw data obtained in the trials using a replicate plot for each quality mark as shown in Figure 2. Since the variation in the trials with replicated parameter set (Parameter Set Index 15 in Table 1) in all the plots showed in Figure 2, for both quality marks, are much smaller than the variation in the entire investigation series, it was possible to conclude that the replicate error would not complicate the data analysis, that means, a good model could be obtained for any wavelength using any quality mark.

The second analysis was based in the evaluation of the plots of the resulting average roughness versus average ablated depth for each trial number and for each kind of wavelength used in the trials. These plots are shown in the Figure 3 . It is possible to observe in these plots that the average roughness is much smaller in the experiments using Ultraviolet than in the experiments using Green and Infrared wavelength. The average Ra and the minimum Ra for the experiments are, respectively, $4.37 \mu \mathrm{m}$ and $1.69 \mu \mathrm{m}$ using Green, $7.02 \mu \mathrm{m}$ and $1.95 \mu \mathrm{m}$ using Infrared and, 0,98 $\mu \mathrm{m}$ and $0,58 \mu \mathrm{m}$ using Ultraviolet wavelength. On the other hand, it is possible to observe that the ablated depth is much bigger in the experiments using Infrared compared to the experiments using Green and Ultraviolet wavelength. The average ablated depth for the experiments are $462.02 \mu \mathrm{m}$ for the Infrared, $152.97 \mu \mathrm{m}$ for the Green and $39.86 \mu \mathrm{m}$ for the Ultraviolet. Since the optimum set of process parameters should achieve, as first criteria, the best surface roughness, the Ultraviolet wavelength was the one that better reached the demanded roughness for the wished biomimetic surfaces and was therefore selected for the next analysis and optimisation step. 

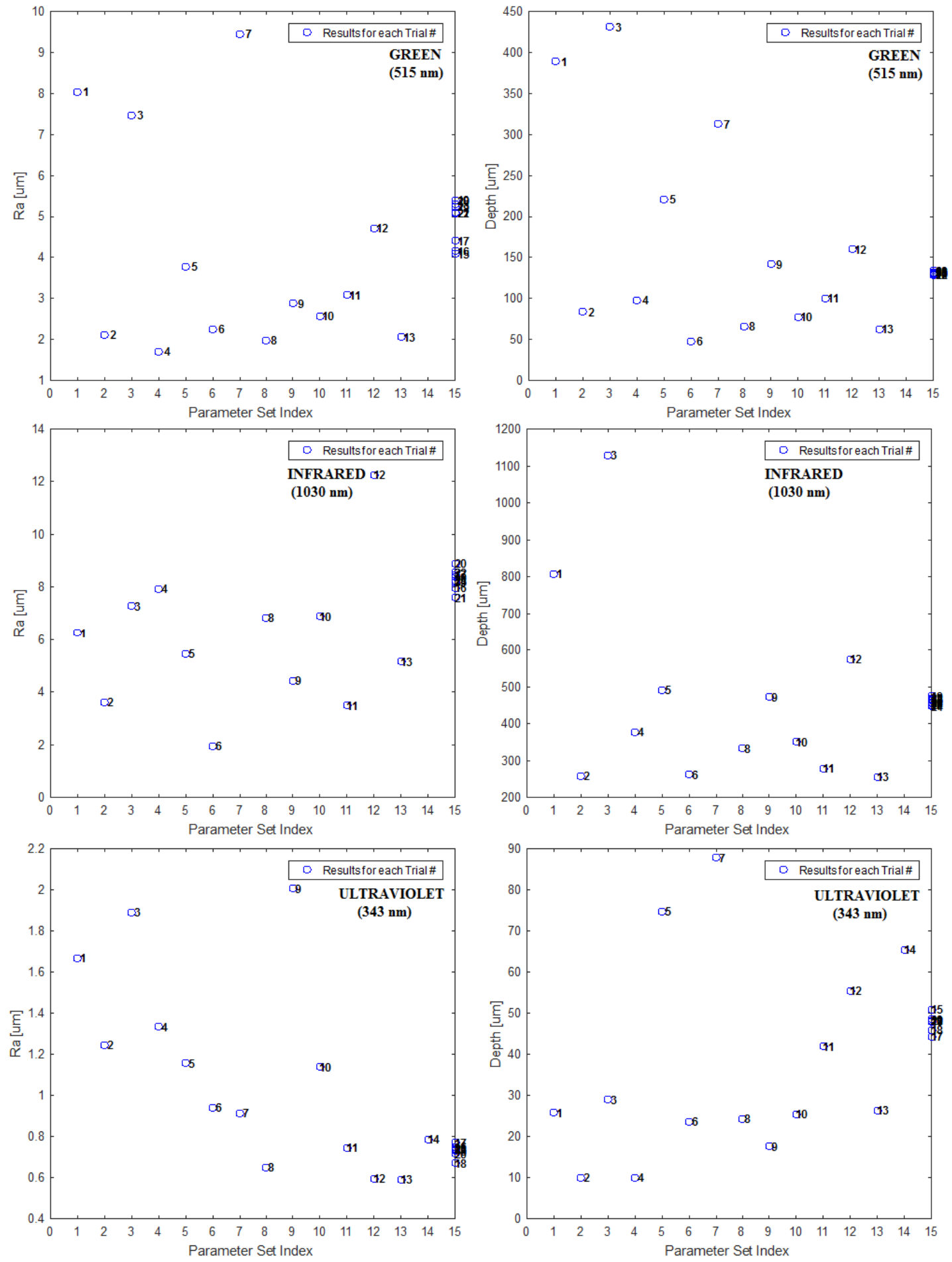

Figure 2. Replicate plots showing the average roughness (Ra $[\mu \mathrm{m}])$ and average depth (Depth $[\mu \mathrm{m}])$ for each Trial Number\# using the different wavelength laser light (Green, Infrared and Ultraviolet). 

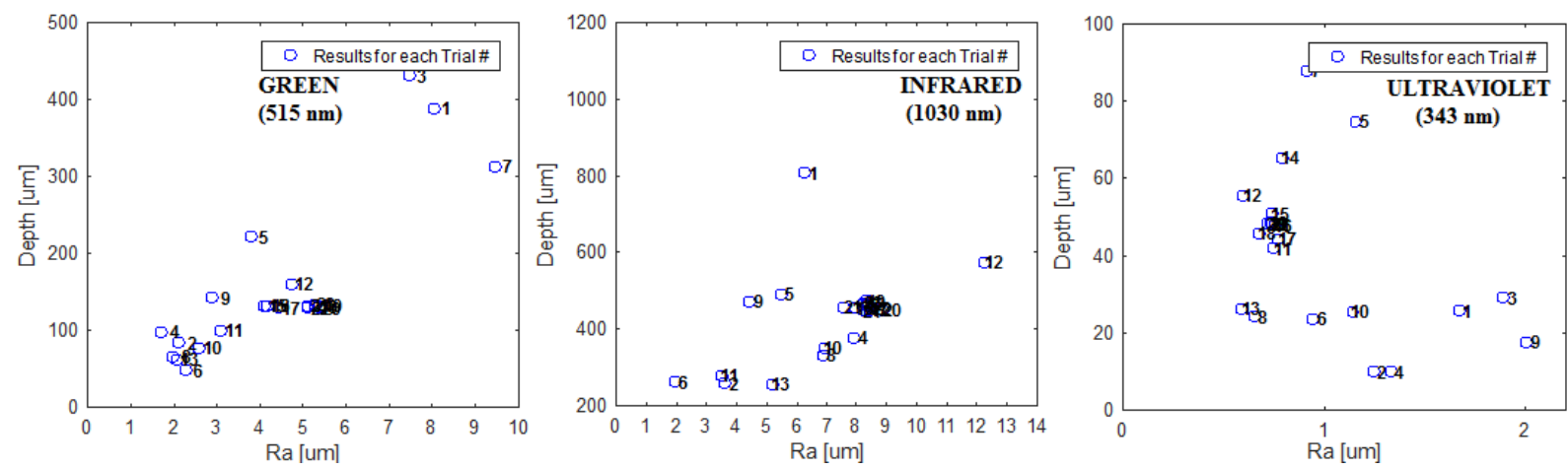

Figure 3. Plots showing average roughness $(\mathrm{Ra}[\mu \mathrm{m}])$ versus average ablated depth (Depth $[\mu \mathrm{m}])$ for each Trial Number\# using the different wavelength laser light (Green, Infrared and Ultraviolet).

The next step was, using linear regression to fit the data of the Ultraviolet experiments, to obtain a consistent process model. It started with a try of a model with only linear terms and the interactions between each predictor but the results were not acceptable with $\mathrm{R}^{2}$ smaller than 0.4 . It was included then the possibility of squared terms for the predictors in the model. Consistent results were then obtained for the process model of both quality marks. The process model considering the roughness as quality mark have a $\mathrm{R}^{2}=0.93$ and a $\mathrm{p}$-value $=1.31 \mathrm{e}-05$ and the process model considering the ablated depth as quality mark have a $\mathrm{R}^{2}=0.89$ and a $\mathrm{p}$-value $=2.26 \mathrm{e}-04$. Plots of the scaled and centered regression coefficients of the obtained model for roughness and ablated depth are shown in the Figure 4.

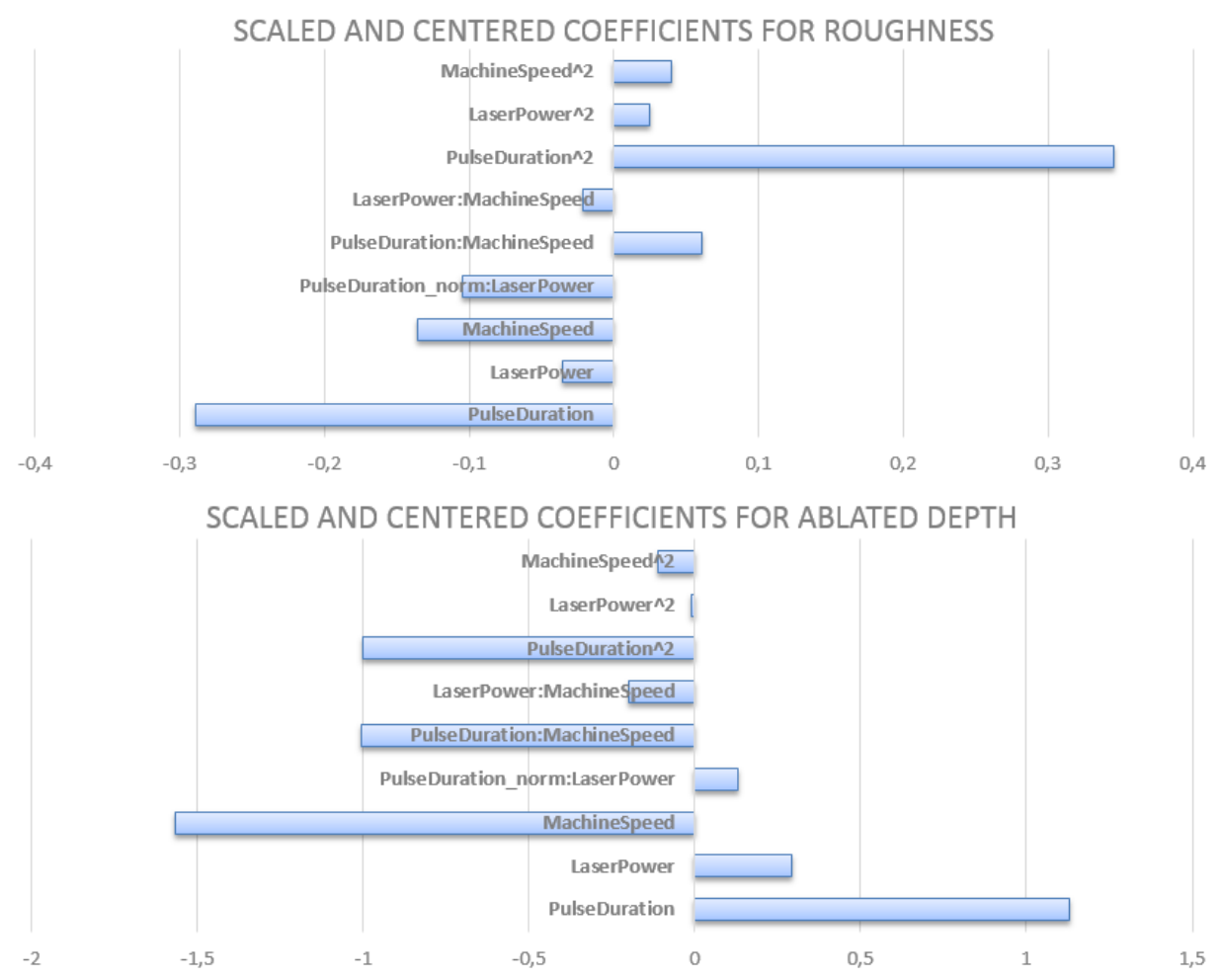

Figure 4. Regression coefficients for roughness and ablated depth models. 
Analysing the plots in the Figure 4, it is possible to notice that the pulse duration is the main parameter that affects the roughness, followed by the machining speed, been the laser power the parameter with the smaller effect. In relation to the ablated depth, the machining speed seems to have the bigger influence, but if one takes into account the sum of the linear and quadratic coefficients of the pulse duration, it becomes the parameter with the greatest influence. Also for the ablated depth, the laser power has the smaller influence between the three parameters.

Slice plots were then produced to visualize the effects of each predictor on the model and they are shown in Figure 5. In the range of values considered in the experiments, while machining speed and laser power have an almost linear influence on achievable roughness, laser pulse duration has a quadratic influence in relation to a global minimum on the surface roughness result. For the ablated depth, machining speed and laser power have an almost linear influence while laser pulse duration has a quadratic influence in relation to a global maximum.

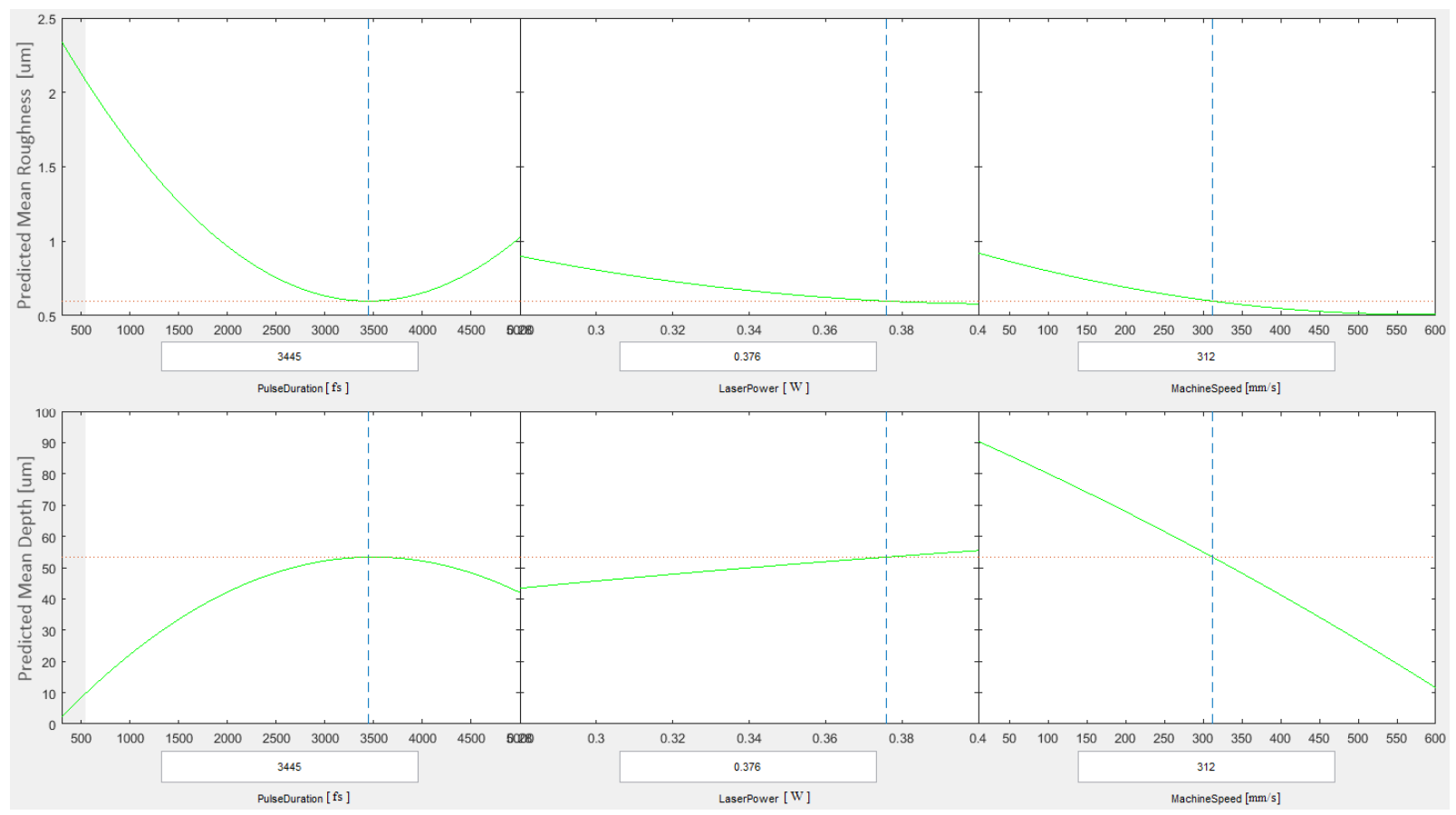

Figure 5. Slice plots showing the effect of each predictor and the optimised process parameters obtained (dashed lines).

In order to obtain optimised process parameters for achieving best surface roughness and, as second criteria, for machining time to be reduced to a minimum, it was found for the laser pulse duration the value of $3445 \mathrm{fs}$ that minimizes the roughness and maximizes the ablated depth. For the laser power, the maximum value $0.376 \mathrm{~W}$, related to the notation " $+1 "$, was the optimized value for this parameter since it minimizes the roughness and maximizes the ablated depth. In relation to the machining speed, the optimized value found was $312 \mathrm{~mm} / \mathrm{s}$. These optimised values are shown in the Figure 5 in the form of dashed lines. 


\section{CONCLUSION}

The trials with different parameter sets and replications showed that the process is stable, making it possible to obtain a process model based on the three selected predictors using the measured roughness and ablated depth as quality mark, been this model essential to understand the effects of these predictors in the process and to optimize their values in order to have a process able to manufacture biomimetic surfaces with micro and nano scales.

Further work will involve testing the parameters obtained manufacturing biomimetic surfaces like house fly eye and shark skin structures.

\section{REFERENCES}

[1] Liu, X., Du, D. and Mourou, G., "Laser ablation and micromachining with ultrashort laser pulses," IEEE journal of quantum electronics 33(10), 1706-1716 (1997).

[2] Breitling, D., Ruf, A. and Dausinger F., "Fundamental aspects in machining of metals with short and ultrashort laser pulses", Proc. SPIE 5339, 49-63 (2004).

[3] Meijer, J., Du, K., Gillner, A., Hoffmann, D., Kovalenko, V. S., Masuzawa, T. and Schulz, W., "Laser machining by short and ultrashort pulses, state of the art and new opportunities in the age of the photons," CIRP Annals-Manufacturing Technology 51(2), 531-550 (2002).

[4] Jeon, Y. and Choon, M. L. "Current research trend on laser assisted machining," International journal of precision engineering and manufacturing 13(2), 311-317 (2012).

[5] Cheng, J., Liu, C. S., Shang, S., Liu, D., Perrie, W., Dearden, G., and Watkins, K., "A review of ultrafast laser materials micromachining." Optics \& Laser Technology 46, 88-102 (2013).

[6] Petkov, P. V., Scholz, S. G., and Dimov, S. S., " Strategies for material removal in laser milling, " 4M 2008 Fourth International Conference on Multi-Material Micro Manufacture Proceedings, Cardiff, 249-252(2008).

[7] Pham, D. T., Dimov, S. S. and Petkov, P. V., "Laser milling of ceramic components." International Journal of Machine Tools and Manufacture 47(3), 618-626 (2007).

[8] Pham, D. T., Dimov, S. S., Ji, C., Petkov, P. V., and Dobrev, T., "Laser milling as a 'rapid'micromanufacturing process, " Proceedings of the Institution of Mechanical Engineers, Part B: Journal of Engineering Manufacture, 218(1), 1-7 (2004).

[9] Abbott, S. J., and Gaskell, P. H., "Mass production of bio-inspired structured surfaces." Proceedings of the Institution of Mechanical Engineers, Part C: Journal of Mechanical Engineering Science 221(10), 1181-1191 (2007).

[10] Scholz, S. G., Griffiths, C. A., Dimov, S. S., Brousseau, E. B., Lalev, G. and Petkov, P., "Manufacturing routes for replicating micro and nano surface structures with bio-mimetic applications." CIRP Journal of Manufacturing Science and Technology, 4(4), 347-356 (2011).

[11] Neuenschwander, B., Jaeggi, B., Schmid, M., Rouffiange, V. and Martin, P. E., "Optimization of the volume ablation rate for metals at different laser pulse-durations from ps to fs," Proc. SPIE 8243, 824307 (2012).

[12] Optec, Laser Micromachining Systems, http://www.optec.be/products/short-pulse-dpss-lasers/sp450-to-kit/ (28 November 2016).

[13]FRT GmbH, The Measure of Precision, http://www.frt-gmbh.com/en/microprof-100.aspx (28 November 2016).

[14] Mathworks, Statistics and Machine Learning Toolbox, http://www.mathworks.com/help/stats/index.html (28 November 2016). 